\title{
Wisdom of experience or faculty of reason: Ischemic mitral regurgitation trials - Cardiothoracic Surgical Trials Network and beyond
}

Robert E. Michler, MD

Ischemic mitral regurgitation (IMR) is a clinical condition that deserves our attention for a number of important reasons, including the prevalence of IMR has been steadily increasing; the clinical consequences of IMR are significant; and our improved understanding of the interaction between IMR severity, left ventricular (LV) geometry, and LV function have led to preferred treatment options and improved clinical results. ${ }^{1-4}$

IMR is a consequence of regional wall motion abnormalities induced by myocardial ischemia and infarction. The interaction between postinfarction/ischemic regional wall motion abnormalities, papillary muscle displacement, leaflet tethering, reduced closing forces, and annular dilatation result in MR. ${ }^{5}$ The mitral valve (MV) leaflets appear normal.

Adverse LV remodeling develops in approximately $50 \%$ of patients after a myocardial infarction. ${ }^{6,7}$ The presence of any degree of IMR identifies patients with LV dysfunction who have a higher mortality risk than those without IMR. ${ }^{6}$ This fact alone remains the primary driver for considering a MV procedure in patients with IMR requiring coronary artery bypass grafting (CABG). To date, no IMR surgical trial has demonstrated improved survival following IMR resolution.

The appropriate surgical management of IMR at the time of CABG begins with a thorough understanding and preferably, serial evaluation of IMR severity. My current decision algorithm for managing any degree of IMR focuses on 5 preoperative factors that help determine the surgical plan. In conjunction with echocardiography and cardiac catheterization, cardiac magnetic resonance imaging with gadolinium hyperenhancement can complete a comprehensive analysis and evaluate the degree of LV scar.

From the Departments of Surgery and Cardiothoracic and Vascular Surgery, Montefiore Medical Center, Albert Einstein College of Medicine, New York, NY.

Received for publication Nov 9, 2019; revisions received Nov 9, 2019; accepted for publication Dec 13, 2019.

Address for reprints: Robert E. Michler, MD, Departments of Surgery and Cardiothoracic and Vascular Surgery, Montefiore Medical Center, Albert Einstein College of Medicine, Medical Arts Pavilion 5th Floor, 3400 Bainbridge Ave,

New York, NY 10467 (E-mail: rmichler@montefiore.org).

JTCVS Open 2020;1:12-6

2666-2736

Copyright (C) 2020 by The Authors. Published by Elsevier Inc. on behalf of The American Association for Thoracic Surgery. This is an open access article under the CC BY-NC-ND license (http://creativecommons.org/licenses/by-nc-nd/4.0/).

https://doi.org/10.1016/j.xjon.2019.12.004

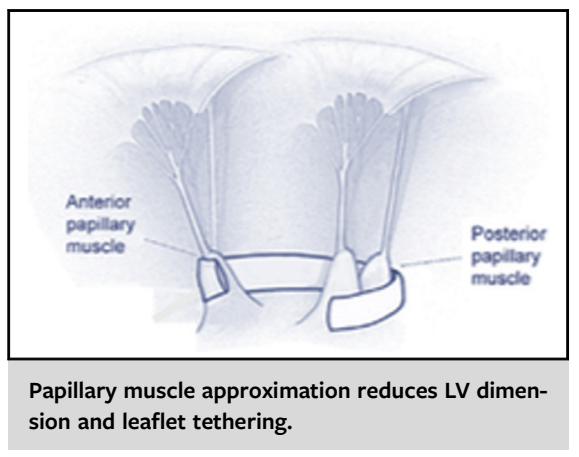

CENTRAL MESSAGE

When determining whether to perform an MV repair procedure or MVR with CABG in ischemic MR, vital considerations include degree of IMR severity, LV dysfunction, LV abnormal geometry, scar, and poor coronary targets.

See Commentary on page 17.

- Severity of MR,

- Severity of LV dysfunction,

- Severity of LV remodeling,

- Presence and extent of LV scar, and

- Quality and distribution of the left circumflex and right coronary circulation.

Mild IMR should be treated with CABG alone because of ample clinical evidence demonstrating the near certain resolution of the IMR. The surgical treatment options differ for moderate and severe IMR and therefore, distinguishing between moderate and severe IMR is critically important. For moderate ischemic disease, the surgical options range from CABG alone to CABG plus an MV repair procedure, and for severe ischemic disease, the surgical options range from $\mathrm{CABG}$ plus an $\mathrm{MV}$ repair procedure to $\mathrm{CABG}$ plus MV replacement. Let us review the evidence to support these options based on IMR severity.

\section{MODERATE IMR}

Historically, some experts have advocated for CABG alone in moderate IMR, expecting improvements in 
regional/global LV function and LV geometry following CABG to lead to a reduction in IMR. ${ }^{8,9}$ Other experts have supported a restrictive mitral annuloplasty (RMA) repair at the time of CABG to address more directly the IMR, expecting to prevent further adverse remodeling and decrease the risk of heart failure. ${ }^{10,11}$

The Cardiothoracic Surgical Trials Network (CTSN) Moderate IMR multicenter randomized trial ${ }^{12,13}$ compared CABG alone with CABG plus RMA in 301 patients with moderate IMR. The primary end point measured was change in end systolic volume index (LVESVI). RMA resulted in a significant reduction over CABG alone in the prevalence of MR at 1 and 2 years and, importantly, no progression to severe MR was seen in the RMA group. ${ }^{13}$ At 1 and 2 years, there was no difference between the 2 groups in change in LVESVI, survival, LV ejection fraction, New York Heart Association functional class, or major adverse cardiac and cerebrovascular events. RMA was associated with a longer hospital stay after surgery, a higher incidence of postoperative supraventricular arrhythmias, and more general neurologic events (eg, metabolic encephalopathy, seizures, transient ischemic attack, and stroke).

In contrast, 2 distinct yet similarly designed randomized clinical trials produced very different results from the CTSN trial. ${ }^{10,11}$ The study by Fattouch and colleagues ${ }^{10}$ and the Randomized Ischemic Mitral Evaluation (RIME) trial $^{11}$ both demonstrated that the addition of a restrictive mitral annuloplasty to CABG resulted in significant improvements in LV reverse remodeling, LV ejection fraction, MR grade and New York Heart Association functional class. Specifically, Fattouch and colleagues ${ }^{10}$ randomly assigned 102 patients and demonstrated that the addition of RMA significantly reduced LV end systolic dimension (LVESd) and LV end diastolic dimension (LVEDd) over CABG alone. The RIME trial ${ }^{11}$ randomly assigned 73 patients and demonstrated a dramatic $28 \%$ reduction in LVESVI over the baseline mean of $78 \mathrm{~mL} / \mathrm{m}^{2}$. The importance of achieving LV size reduction lies in the knowledge that LV enlargement is an independent risk for mortality. In part, this is why the CTSN chose LV dimension as the primary end point for its trial.

Why were the clinical outcomes of the CTSN trial unable to achieve the benefits seen in the Fattouch and colleagues ${ }^{10}$ and RIME $^{11}$ randomized clinical trials? There are several points to consider. First, in the CTSN trial, irrespective of treatment arm, patients with resolution of IMR had greater reverse remodeling and better wall motion scores than those who did not. ${ }^{13}$ This point is noteworthy because it provides evidence that many patients in both arms of the CTSN trial had viable myocardium and minimal LV scar. Improvements in global and regional wall motion as well as reverse LV remodeling following CABG alone are indicative of viable myocardium. ${ }^{9,14}$ In such patients, revascularization alone would undoubtedly result in a reduction in MR. As Penicka and colleagues ${ }^{8}$ demonstrated in a series of patients with moderate IMR who underwent CABG alone, resolution of IMR following surgery was associated at baseline with more viable segments and less LV dysynchrony.

Second, patients in the CTSN trial experienced substantially lower rates of baseline prior myocardial infarction and therefore, less LV scar than in the Fattouch and colleagues ${ }^{10}$ and RIME $^{11}$ studies. Patients with LV scar are less likely to experience wall motion improvement from revascularization and consequently, such patients may be better suited to the addition of RMA as a treatment for IMR.

Third, baseline LV size was significantly smaller in the CTSN trial compared with the Fattouch and colleagues ${ }^{10}$ and RIME $^{11}$ trials. This fact alone may have favored patients in the Fattouch and colleagues ${ }^{10}$ and RIME $^{11}$ trials who received a restrictive mitral annuloplasty, especially in the presence of scar, because CABG alone in these patients would be less likely to result in sufficient improvement in LV wall motion and LV size reduction, precisely those factors that influence IMR resolution.

Fourth, significant reverse remodeling was achieved in the Fattouch and colleagues ${ }^{10}$ and RIME $^{11}$ trials with CABG plus RMA compared with CABG alone. In the RIME trial, ${ }^{11}$ a $28 \%$ reduction in LV size from baseline (baseline mean, $78 \mathrm{~mL} / \mathrm{m}^{2}$ ) was achieved with CABG plus RMA, whereas in the CTSN trial patients who received the combined procedure saw only a $9 \%$ reduction in $\mathrm{LV}$ size from baseline (baseline mean, $57 \mathrm{~mL} / \mathrm{m}^{2}$ ). To underscore this important point, patients enrolled in the CTSN trial had smaller ventricles at baseline and as the evidence implies, more viable myocardium-expressly the clinical substrate that is likely to benefit the most from CABG alone when seeking to achieve IMR reduction. Unfortunately, none among these 3 clinical trials required preoperative evaluation of myocardial viability, which may have helped in our understanding of the subsequent clinical outcome. It is known that viability assessment can help predict the effectiveness of revascularization in specific patient populations and must be considered in this setting. ${ }^{15}$

The lessons learned from these randomized trials in moderate IMR emphasize the fact that individual treatment decisions require balancing the risks of adverse perioperative events against the benefits of a lower incidence of postoperative IMR. One surgical therapy does not fit all clinical scenarios of LV geometry and function. Effective revascularization, as reflected in improved regional and global LV function, plays an important role in reducing moderate IMR independent of restrictive annuloplasty repair. In other clinical settings, the anticipated low likelihood of generating significant functional improvement and reverse remodeling from $\mathrm{CABG}$ should lead to the performance of a restrictive mitral annuloplasty reparative procedure. Such circumstances include patients with 
documented scar or basal aneurysm/dyskinesia in the inferior-posterior-lateral LV, larger ventricles (LVESVI $>50 \mathrm{~mL} / \mathrm{m}^{2}$ and LVEDd $>55 \mathrm{~mm}$ ), and poor coronary targets in the circumflex/right coronary distributions because of the reduced likelihood that revascularization will provide significant enhancement of LV contractility and $L V$ reverse remodeling.

Whether a restrictive mitral annuloplasty repair together with CABG in moderate IMR will predictably benefit patients with LV enlargement, poor coronary targets, or baseline inferior-posterior-lateral wall motion abnormalities considered to be scar remains unknown. Until such evidence is available, restrictive mitral annuloplasty should be considered an essential part of the surgical armamentarium for these patients.

\section{SEVERE IMR}

The optimal surgical management of severe IMR is debatable and in evolution. Some experts advocate a chordal-sparing MV replacement, whereas other experts support a restrictive mitral annuloplasty repair alone or in combination with a papillary muscle approximation (PMA) procedure to reduce LV dimension. ${ }^{16-22}$ Although a MV repair procedure is associated with lower operative morbidity and mortality, as well as the presumed benefit of maintaining LV systolic function by preserving the subvalvular apparatus, the 1-year MR recurrence rate with RMA alone can be as high as $30 \%$ to $40 \%{ }^{16-18,23} \mathrm{MV}$ replacement, although more effective and durable in addressing IMR, is associated with greater operative risk, and greater long-term risks such as thromboembolism, valve deterioration, and endocarditis. ${ }^{19-22}$

The CTSN sought to address this controversy by comparing chordal-sparing MV replacement to restrictive mitral annuloplasty in 251 patients with severe IMR. ${ }^{16,17}$ Chordal-sparing MV replacement resulted in a significant reduction over RMA in the prevalence of recurrent/persistent MR at 1 year $(2.3 \%$ vs $32.6 \%)$ and 2 years $(3.8 \%$ vs $58.8 \%$ ). At 1 and 2 years, there was no difference between the 2 groups in the degree of LV reverse remodeling, LV ejection fraction, survival, or major adverse cardiac and cerebrovascular events. Although the rate of serious adverse events and overall hospital readmissions were comparable, patients undergoing RMA had more frequent events related to heart failure and cardiovascular readmissions at 2 years. These data support the use of MV replacement in the setting of severe IMR for patients with similar clinical characteristics to those enrolled in the CTSN trial. As with all clinical studies, the implications and generalizability of the study require further examination.

Critics of the CTSN trial contend that the high prevalence of persistent/recurrent MR with RMA alone was related to trial design and surgical technique deficiencies in the conduct of the CTSN trial. ${ }^{4,18}$ For example, only $75 \%$ of patients received concomitant CABG surgery and hence, $25 \%$ of CTSN patients enrolled had a clinical substrate incapable of benefiting from the ability of a revascularization procedure to produce wall motion improvement and IMR reduction. Moreover, critics of the CTSN trial cite insufficient undersizing of the mitral annulus with RMA, which limited the ability of the leaflets to form an improved zone of coaptation to reduce tenting height and tenting area. ${ }^{18}$ In contrast to the CTSN trial, Nappi and colleagues, ${ }^{18}$ in the Papillary Muscle Approximation Randomized Trial, achieved a 2-year prevalence of recurrent/ persistent MR with RMA alone of only $13.2 \%$, which the authors claim was the result of more effective annular reduction as seen by a greater mean difference between annular size and ring size compared with the CTSN trial. ${ }^{18}$

Indeed, the strategy of overcorrection in restrictive annuloplasty (ie, the tighter, the better) has been advocated for a number of years by many respected surgeons. But, can such an overcorrection strategy reliably produce consistent and durable IMR resolution? Or, is there something more at play that must be considered to achieve durable IMR resolution? An evolving sentiment among experts is that an overcorrection strategy with RMA alone will not reliably produce long-term IMR reduction, especially because RMA alone does not consider or therapeutically address LV enlargement and its influence on tethering forces. It is intellectually far reaching and probably wishful thinking to expect that a redesign of the prosthetic annuloplasty ring could alter LV anatomy sufficiently to reliably reverse adverse LV remodeling.

Logic suggests that the best candidates for an RMA-alone procedure are those patients with a normal to mildly enlarged left ventricle and in particular, those ventricles that can achieve reverse remodeling as a result of simultaneous revascularization. Larger and scared ventricles represent clinical scenarios in which it is unlikely to expect a reliable and durable reduction in IMR from an annular reduction procedure alone. The evidence for this line of thinking in severe IMR arises from multiple observations, including restrictive mitral annuloplasty does not reliably prevent persistent or recurrent IMR, overcorrected restrictive mitral annuloplasty does lead to mitral stenosis, and overcorrection using severely undersized annuloplasty rings is associated with exacerbated leaflet tethering. ${ }^{16,17,23}$

This latter point deserves further explanation. Recent post hoc analysis by the CTSN authors highlight the importance of understanding the influence of restrictive mitral annuloplasty on the spatial relationship between the left ventricle and the mitral annulus. ${ }^{23}$ Because the anterior leaflet is attached to the fibrous trigones, it is the posterior leaflet that is primarily affected by restrictive mitral annuloplasty, and mitral annular area is reduced by reducing anterior-posterior dimension. If the posterior-inferiorlateral wall of the left ventricle remains displaced because 
of scar or deficient reverse remodeling, overcorrection RMA produces an increase in the geometric distance mismatch between the displaced ischemic papillary muscles and the mitral annulus, resulting in an exacerbation of posterior leaflet tethering. In other words, overcorrection of restrictive mitral annuloplasty exacerbates tethering of the posterior leaflet when the ischemic papillary muscles remain laterally and apically displaced relative to the mitral annulus.

With this in mind, the CTSN authors evaluated the ratio of LVESd to prosthetic annuloplasty ring size and its ability to predict the risk of persistent or recurrent MR following restrictive mitral annuloplasty. ${ }^{23}$ The authors identified an LVESd to ring size ratio $\geq 2$ to be associated with an increased risk of persistent or recurrent IMR. Thus, increased tethering between the papillary muscle and leaflet edge can be produced by overcorrection of the annular dimension. Therefore, RMA is a necessary, but insufficient procedure in the treatment of severe IMR. Surgeons can readily assess this issue in their preoperative evaluation and plan appropriately in the operating room. But, will this strategy be sufficient to ensure the elimination of IMR in the operating room and provide a durable result with RMA alone? Unlikely, because it does not address LV enlargement and papillary muscle displacement.

A logical therapeutic adjuvant to an appropriately sized annular correction with RMA would be to surgically reduce LVESd. Reducing LVESd would have the opposite effect of annular overcorrection because it would bring the papillary muscle closer to the mitral annulus and thus decrease leaflet tethering forces. Think of it this way: Reanimating ischemic muscle by coronary revascularization will improve regional wall motion and reduce LVESd and IMR. Because one cannot preoperatively reliably predict the degree of regional wall motion improvement and reverse remodeling achievable with revascularization alone (and hence, the likelihood of reducing LVESd and IMR), an additional ventricular surgical procedure such as PMA may be a necessary surgical complement to an appropriately sized restrictive mitral annuloplasty. ${ }^{4,18,23}$

In support of this notion is recent work by Nappi and colleagues. ${ }^{24}$ They evaluate preoperative echocardiograms in 96 patients who underwent CABG plus restrictive mitral annuloplasty versus CABG plus restrictive mitral annuloplasty and PMA in the Papillary Muscle Approximation Randomized Trial for severe IMR. They sought to determine whether any baseline echocardiographic parameter served as a marker for IMR recurrence.

Of the 48 patients who underwent CABG plus RMA and PMA, 37 survived to 5 years and were available for matched clinical and echocardiographic evaluation. At baseline, 21 patients had moderate-severe IMR and 16 had severe IMR. Of the 37 patients, 31 left the operating room with none/trace IMR and 6 with mild IMR. By 5 years, 27
$(73 \%)$ had none/trace IMR, $9(24.3 \%)$ had moderate IMR, $1(2.7 \%)$ had moderate-severe IMR, and 0 patients had severe IMR. Therefore, at 5 years, a very durable reduction in IMR severity was achieved with RMA plus PMA and no patients experienced severe IMR.

A relatively small number of patients were enrolled, yet these results for RMA plus PMA surpass other published reports of late IMR recurrence rates for severe IMR treated by RMA alone. In addition, the results exceed the IMR recurrence rate of RMA alone in the other arm of the Papillary Muscle Approximation Randomized Trial. In the RMA alone arm at 5 years, the 34 surviving patients experienced a $55.9 \%$ prevalence of moderate or severe IMR, despite having experienced a recurrence rate of $13.2 \%$ at 2 years (38 patients). Although delayed in time, the data for RMA alone are consistent with the data from the CTSN trial and other published reports demonstrating a significant prevalence of late IMR with RMA alone.

In the Papillary Muscle Approximation Randomized Trial, patients who underwent CABG plus RMA and PMA experienced a significant improvement over baseline in LV ejection fraction, reverse LV remodeling, MV tenting height, MV tenting area, and interpapillary muscle distance. These improvements were further magnified when the 27 patients with no/trace IMR were compared with the $10 \mathrm{pa}-$ tients with moderate or greater IMR.

Statistical modeling by Nappi and colleagues ${ }^{18,24}$ identified preoperative factors associated with persistent or recurrent IMR following CABG plus RMA and PMA. The predictive baseline markers included MV tenting area $>3.1 \mathrm{~cm}^{2}$, LVEDd $>64 \mathrm{~mm}$, and LVESd $>54 \mathrm{~mm}$. These findings make intuitive sense because these markers represent larger ischemic remodeled ventricles, predictably those ventricles least likely to recover sufficient regional and global function to significantly reverse remodel following CABG.

Decision making for patients with severe IMR is enhanced by preoperative identification of those patients most likely to have an improvement in regional wall motion and global LV function following CABG. Preoperative evaluation of myocardial viability, echocardiographic assessment of regional and global LV systolic function, and echocardiographic assessment of MV tethering parameters can help in the planning for which mitral valve procedure to perform in severe IMR. CMR imaging with gadolinium hyperenhancement is an appropriate tool when echocardiography or radionuclide imaging are equivocal or in patients suspected to have LV scar and hibernating myocardium.

Therefore, this author believes there is a role for both an MV repair procedure and MV replacement in patients with severe IMR. The principle parameters that favor a replacement over an MV repair procedure include patients with poor coronary targets in the circumflex/right coronary distributions (ie, reduced likelihood that $\mathrm{CABG}$ will provide 
significant enhancement of LV contractility and reverse remodeling), scar or basal aneurysm/dyskinesia in the inferior-posterior-lateral LV, large ventricles (LVESVI $>70 \mathrm{~mL} / \mathrm{m}^{2}$ or LVESd $>55 \mathrm{~mm}$ or LVEDd $>65 \mathrm{~mm}$ ), and an MV tenting area $>3 \mathrm{~cm}^{2}$.

Recognition that a durable repair is not only possible, but also likely following CABG plus RMA and PMA in patients presenting with a nonseverely dilated LV, low MV tenting area, and good coronary targets should influence surgeons to strongly consider MV repair therapy in patients with severe IMR.

\section{Conflict of Interest Statement}

The authors reported no conflicts of interest.

The Journal policy requires editors and reviewers to disclose conflicts of interest and to decline handling or reviewing manuscripts for which they may have a conflict of interest. The editors and reviewers of this article have no conflicts of interest.

\section{References}

1. Baumgartner H, Falk V, Bax JJ, De Bonis M, Hamm C, Holm PJ, et al. 2017 ESC/ EACTS guidelines for the management of valvular heart disease. Eur Heart J. 2017;38:2739-91.

2. de Marchena E, Badiye A, Robalino G, Junttila J, Atapattu S, Nakamura M, et al. Respective prevalence of the different Carpentier classes of mitral regurgitation: a stepping stone for future therapeutic research and development. J Card Surg. 2011;26:385-92.

3. Nappi F, Avatar Singh SS, Santana O, Mihos CG. Functional mitral regurgitation: an overview for surgical management framework. J Thorac Dis. 2018;10:4540-55.

4. Michler RE. Surgical management of moderate ischemic mitral regurgitation at the time of coronary artery bypass grafting remains controversial. J Thorac Cardiovasc Surg. 2018;156:1498-500.

5. Grigioni F, Detaint D, Avierinos JF, Scott C, Tajik J, Enriquez-Sarano M. Contribution of ischemic mitral regurgitation to congestive heart failure after myocardial infarction. J Am Coll Cardiol. 2005;45:260-7.

6. Bursi F, Enriquez-Sarano M, Nkomo VT, Jacobsen SJ, Weston SA, Meverden RA, et al. Heart failure and death after myocardial infarction in the community: the emerging role of mitral regurgitation. Circulation. 2005;111: 295-301.

7. Perez de Isla L, Zamorano J, Quezada M, Almería C, Rodrigo JL, Serra V, et al. Functional mitral regurgitation after a first non-ST-segment elevation acute coronary syndrome: contribution to congestive heart failure. Eur Heart J. 2007;28: 2866-72.

8. Penicka M, Linkova H, Lang O, Fojt R, Kocka V, Vanderheyden M, et al. Predictors of improvement of unrepaired moderate ischemic mitral regurgitation in patients undergoing elective isolated coronary artery bypass graft surgery. Circulation. 2009;120:1474-81.

9. Roshanali F, Mandegar MH, Yousefnia MA, Alaeddini F, Wann S. Low-dose dobutamine stress echocardiography to predict reversibility of mitral regurgitation with CABG. Echocardiography. 2006;23:31-7.

10. Fattouch K, Guccione F, Sampognaro R, Panzarella G, Corrado E, Navarra E, et al. Efficacy of adding mitral valve restrictive annuloplasty to coronary artery bypass grafting in patients with moderate ischemic mitral valve regurgitation: a randomized trial. J Thorac Cardiovasc Surg. 2009;138:278-85.

11. Chan KM, Punjabi PP, Flather M, Wage R, Symmonds K, Roussin I, et al. Coronary artery bypass surgery with or without mitral valve annuloplasty in moderate functional ischemic mitral regurgitation: final results of the randomized ischemic mitral evaluation (RIME) trial. Circulation. 2012; 126:2502-10.

12. Smith PK, Puskas JD, Ascheim DD, Voisine P, Gelijns AC, Moskowitz AJ, et al. Surgical treatment of moderate ischemic mitral regurgitation. $N$ Engl J Med. 2014;371:2178-88.

13. Michler RE, Smith PK, Parides MK, Ailawadi G, Thourani V, Moskowitz AJ, et al. Two-year outcomes following surgical treatment of moderate ischemic mitral regurgitation. $N$ Engl J Med. 2016;374:1932-41.

14. Sundt TM. Surgery for ischemic mitral regurgitation. N Engl J Med. 2014;371: 2228-9.

15. Meluzín J, Cerný J, Frélich M, Stetka F, Spinarová L, Popelová J, et al. Prognostic value of the amount of dysfunctional but viable myocardium in revascularized patients with coronary artery disease and left ventricular dysfunction. Investigators of this Multicenter Study. J Am Coll Cardiol. 1998; 32:912-20.

16. Acker MA, Parides MK, Perrault LP, Moskowitz AJ, Gelijns AC, Voisine P, et al. Mitral-valve repair versus replacement for severe ischemic mitral regurgitation. N Engl J Med. 2014;370:23-32.

17. Goldstein D, Moskowitz AJ, Geijins AC, Ailawadi G, Parides MK, Perrault LP, et al. Two-year outcomes of surgical treatment in severe ischemic mitral regurgitation. N Engl J Med. 2016;374:344-53.

18. Nappi F, Lusini M, Spadaccio C, Nenna A, Covino E, Acar C, et al. Papillary muscle approximation versus restrictive annuloplasty alone for severe ischemic mitral regurgitation: a randomized trial. J Am Coll Cardiol. 2016; 24:2334-46.

19. Gillinov AM, Wierup PN, Blackstone EH, Bishay ES, Cosgrove DM, White J, et al. Is repair preferable to replacement for ischemic mitral regurgitation? J Thorac Cardiovasc Surg. 2001;122:1125-41.

20. Reece TB, Tribble CG, Ellman PI, Maxey TS, Woodford RL, Dimeling GM, et al. Mitral repair is superior to replacement when associated with coronary artery disease. Ann Surg. 2004;239:671-5.

21. Al-Radi OO, Austin PC, Tu JV, David TE, Yau TM. Mitral repair versus replacement for ischemic mitral regurgitation. Ann Thorac Surg. 2005;79:1260-7.

22. Vassileva CM, Boley T, Markwell S, Hazelrigg S. Meta-analysis of short-term and long-term survival following repair versus replacement for ischemic mitral regurgitation. Eur J Cardiothorac Surg. 2011;39:295-303.

23. Capoulade R, Zeng X, Overbey JR, Ailawadi G, Alexander JH, Ascheim D, et al. Impact of left ventricular to mitral valve ring mismatch on recurrent ischemic mitral regurgitation after ring annuloplasty. Circulation. 2016;134:1247-56.

24. Nappi F, Lusini M, Avtaar Singh SS, Santana O, Chello M, Mihos CG. Risk of ischemic mitral regurgitation recurrence after combined valvular and subvalvular repair. Ann Thorac Surg. 2019;108:536-43. 